\title{
¿Cuánta soya, grasa y sal/sodio contienen los chorizos comerciales?
}

\author{
How much soy, fat, and salt/sodium do commercial sausages contain? \\ Vania Palma-González, Samantha J. Cadena-Ramírez ${ }^{b}$,Emili X. Montiel-Resendis ${ }^{c}$, Darien \\ Ramírez-Martínez ${ }^{d}$, Abigail Trejo-Peña ${ }^{e}$, Quinatzin Y. Zafra-Rojas ${ }^{f}$,Esther Ramírez-Moreno ${ }^{g}$
}

\begin{abstract}
:
Chorizo is considered a processed meat product, blanched or matured, stuffed, made from meat and fat from slaughter animals. The objective of this study was to evaluate the amount of soy, fat and salt contained in commercial sausages, considering the regulations that govern these products. A search for chorizos was carried out in supermarkets located in Mexico City, State of Mexico and Hidalgo. The labeling of 12 chorizo brands was analyzed and compared, considering $100 \mathrm{~g}$ of product, taking into account the energy, proteins, carbohydrates, fats, fiber and sodium provided by these products. The commercial chorizos evaluated presented amounts of fat ( 0.33 to $13.8 \%$ ) and sodium (between 1.3 and $10 \mathrm{~g}$ ). According to the provisions of NOM-051-SCFI / SSA1-2010, products that exceed the content $>10 \%$ of saturated fat and $>300 \mathrm{mg}$ of sodium must include warning stamps "Saturated fat excess" and "Sodium excess ". For its part, the amount of soy was not reported in all the products evaluated, however, if the amount of fiber reported in their nutritional table, which could justify the vegetable content from soy in the sausages, which presented amounts from 0.33 to $4.1 \%$
\end{abstract}

Keywords:

Chorizo, nutritional composition, soy, fat, salt

\section{Resumen:}

El chorizo es considerado como un producto cárnico procesado, escaldado o madurado, embutido, elaborado con base de carne y grasa de animales de abasto. El objetivo del presente estudio fue evaluar la cantidad de soya, grasa y sal que contienen los chorizos comerciales considerando la normativa que rige estos productos. Se realizó una búsqueda de chorizos en los supermercados situados en la Ciudad de México, Estado de México e Hidalgo. Se analizó y comparó el etiquetado de 12 marcas de chorizo considerando 100 $\mathrm{g}$ de producto tomando en cuenta la energía, proteínas, carbohidratos, grasas, fibra y sodio que aportan estos productos. Los chorizos comerciales evaluados presentaron cantidades de grasa $(0.33$ a $13.8 \%)$ y sodio (entre 1.3 hasta $10 \mathrm{~g})$. De acuerdo a lo establecido en la NOM-051-SCFI/SSA1-2010, los productos que exceden el contenido $>10 \%$ de grasas saturadas y $>300$ mg de sodio se les debe incluir sellos de advertencia "Exceso de grasa saturada" y "Exceso de sodio". Por su parte, la cantidad de soya no fue reportada en todos los productos evaluados, sin embargo, sí, la cantidad de fibra que se reporta en la tabla nutrimental de los mismos, lo que podría justificar el contenido vegetal a partir de soya en los chorizos, los cuales presentaron cantidades de 0.33 a $4.1 \%$.

Palabras Clave:

\footnotetext{
Autor de Correspondencia, Universidad Autónoma del Estado de Hidalgo, México, https://orcid.org/0000-0001-8694-0164, Email: vanish.pg@gmail.com

b Universidad Autónoma del Estado de Hidalgo, México, https://orcid.org/0000-0001-8120-1282, Email: samcadena2912@gmail.com c Universidad Autónoma del Estado de Hidalgo, México, https://orcid.org/0000-0001-9836-1303, Email: emiliiximena@gmail.com d Universidad Autónoma del Estado de Hidalgo, México, https://orcid.org/0000-0003-4510-0649, Email: darienramirezmtz@ gmail.com e Universidad Autónoma del Estado de Hidalgo, México, https://orcid.org/0000-0002-2343-5870, Email: abmi.trejo14@gmail.com f Universidad Autónoma del Estado de Hidalgo, México, https://orcid.org/0000-0002-5295-9972, Email: quinatzin_zafra@uaeh.edu.mx g Universidad Autónoma del Estado de Hidalgo, México, https://orcid.org/0000-0002-9928-8600, Email: esther_ramirez@uaeh.edu.mx
} 


\section{Introducción}

El chorizo es considerado como un producto cárnico procesado, fresco, escaldado o madurado, embutido, elaborado con base en carne y grasa de animales de abasto. La proporción de los ingredientes depende del tipo de chorizo (rojo o verde), de la variedad (almendrado, apiñonado, comercial y especial) y del gusto del productor. Cabe destacar que, existen cuatro ingredientes básicos en la producción del chorizo: carne, grasa, especias y pigmento. La carne es la que se encuentra en mayor proporción, las especias, tales como la pimienta y el comino son las que brindan aroma y sabor, los pigmentos como el pimentón son responsables de brindar color y algunas ocasiones también sabor. ${ }^{1} \mathrm{La}$ grasa, le proporciona humedad y sabor al producto, sin embargo, cuanto más saturada, más rápido es el enranciamiento del chorizo. $^{2}$ De acuerdo a la NOM-122-SSA1-1994 está permitido que estos productos contengan $2 \%$ de proteína asilada de soya.

En términos generales a los chorizos se les puede clasificar en cuatro categorías: de primera o especial (hechos con lomo o jamón); de segunda o categoría industrial, (contienen $50 \%$ de lomo o jamón de cerdo y $50 \%$ de carne de ternera); de tercera (elaborada con $75 \%$ de carne de vacuno y $25 \%$ de cerdo); de cuarta o tipo económico (contiene carne de vacuno, otros tipos de carne o sustitutos de carne $y / o$ adicionadas con grasa de cerdo). ${ }^{4}$ La NOM-145-SSA1-1995, ${ }^{5}$ clasifica al chorizo dentro del grupo de productos cárnicos troceados y curados. La calidad de estos productos también depende del contenido de grasa, ya que esta puede variar entre 20 y $30 \%$ para productos de alta calidad y de hasta $40 \%$ en productos más económicos. ${ }^{6}$ En la norma técnica NTON $03103-16^{7}$ se menciona que, para el chorizo, el valor permitido de grasa total será del $40 \%$, indicando que el contenido de grasa animal deberá ser igual o mayor al $51 \%$ de la grasa total.

El exceso de sal utilizado en estos productos cárnicos es usado para prolongar su vida media, mejorar el sabor, $y$, además, enmascarar sabores amargos que a veces se generan durante los procesos de elaboración industrial. ${ }^{8}$

El objetivo principal de este estudio fue evaluar la cantidad de soya, grasa y sal que contienen los chorizos comerciales considerando la normativa que rige estos productos.

\section{Metodología}

Se realizó una búsqueda de chorizos en los supermercados situados en la Ciudad de México, Estado de México e Hidalgo.

Se analizó y comparó el etiquetado de 12 marcas de chorizo. La información fue procesada utilizando un programa de Microsoft-Excel 2018. Para llevar a cabo una comparación entre las muestras se incluyó la cantidad de energía, proteínas, carbohidratos, grasas, fibra y sodio por cada $100 \mathrm{~g}$ de producto.

\section{Resultados y Discusión}

En la Tabla 1 se observa la composición nutrimental de los diferentes chorizos comerciales evaluados. Los chorizos muestran un contenido de energía entre 203 a $443 \mathrm{kcal} / 100 \mathrm{~g}$, con excepción de los chorizos vegetarianos que contienen un menor aporte de 70 y 327 $\mathrm{kcal} / 100 \mathrm{~g}$. Esta variabilidad en el contenido de energía está relacionada con la cantidad de proteínas $(8-21 \%)$ y grasa $(0.33$ a $13.8 \%)$ que se obtuvo de estos productos. De acuerdo a la NOM-051-SCFI/SSA1-2010, ${ }^{9}$ con excepción de los productos vegetarianos la mayoría supera el límite establecido de energía (mayor a $275 \mathrm{kcal}$ totales) en $100 \mathrm{~g}$ de producto. Por lo tanto, en casi todos los chorizos, los productores deben considerar la leyenda de advertencia "Exceso de calorías" en el etiquetado y en el chorizo Don Chema debería aparecer el etiquetado de "Exceso de grasas saturadas", ya que supera el límite establecido por dicha norma (mayor al $10 \%$ del total de energía proveniente de grasas saturadas).

El contenido de proteína que tuvieron estos productos fue del 8 al $21 \%$, sin embargo, esta no siempre es de origen animal, y pudiera provenir de fuentes vegetales como la soya lo cual permite reducir el costo del producto.

Feiner ${ }^{10}$ señala que es común utilizar otros ingredientes como la soya, gluten de trigo, caseinato de trigo, caseinato, etcétera, para dar un aporte de fibra a los chorizos comerciales, lo que podría explicar la cantidad de este ingrediente en el etiquetado de estos productos. Marin, ${ }^{11}$ menciona que la adición de fibra a productos como el chorizo cerca del $2 \%$ mejora el rendimiento del producto, así como los parámetros de dureza y gomosidad. Al ser un producto cárnico no debería contener fibra, sin embargo, los diferentes chorizos mostraron un contenido de 0.33 a $4.1 \%$ de fibra, lo cual pudiera ser debido al contenido de soya $u$ otros componentes de origen vegetal. 
Por otro lado, es importante mencionar que la sal es el principal ingrediente que se añade a los embutidos; se agrega del 1 al $5 \%$, dependiendo si el producto es fresco o madurado. Esta cantidad de sodio le permitirá al producto una mayor conservación. ${ }^{12}$ Los productos evaluados presentaron cantidades entre 266 y hasta 1260 mg de sodio, por lo que de acuerdo a la NOM-051-
SCFI/SSA1-2010, ${ }^{9}$ todos los productos deberían presentar en su etiquetado el sello de "Exceso de sodio", ya que superan el límite permitido por dicha norma (300 $\mathrm{mg}$ de sodio/100 $\mathrm{g}$ de producto). Generalmente, la cantidad de sodio tan alta es enmascarada con carbohidratos por lo que se pude encontrar un contenido de 1.3 a $10 \mathrm{~g}$ de estos componentes.

Tabla 1. Composición nutrimental de chorizos comerciales consumidos en México g/100 g.

\begin{tabular}{|c|c|c|c|c|c|c|c|}
\hline $\begin{array}{l}\frac{\pi}{0} \\
\sum \\
\sum\end{array}$ & $\begin{array}{l}\frac{0}{0} \\
\circ \stackrel{N}{\bar{*}} \\
\text { 을 }\end{array}$ & 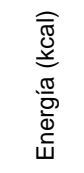 & 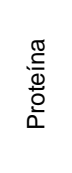 & 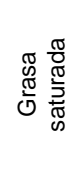 & $\frac{\pi}{\frac{\pi}{2}}$ & 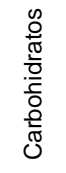 & $\begin{array}{l}\widehat{\widehat{D}} \\
\text { छ } \\
\text { 읗 } \\
\stackrel{\circ}{\infty}\end{array}$ \\
\hline \multirow[t]{2}{*}{ Chata } & De cerdo ranchero & 277 & 13 & 7 & 4 & 9 & 1050 \\
\hline & De soya & 327 & 15.2 & 6.8 & 3.6 & 7.2 & 1004 \\
\hline \multirow[t]{2}{*}{$\mathrm{KIR}$} & Taquero & 333.3 & 11.6 & 2.3 & 0.3 & 1.3 & 680 \\
\hline & Ranchero & 366.6 & 11.3 & 3.3 & 0 & 2.3 & 693.3 \\
\hline \multirow[t]{2}{*}{ Sabori } & Vegetariano & 253.3 & 16.6 & 3.3 & 0 & 10 & 900 \\
\hline & De pavo & 284 & 11 & 6 & 0 & 5 & 841 \\
\hline \multirow[t]{3}{*}{ Chimex } & De cerdo ranchero & 356.6 & 9.6 & 11.3 & 1.6 & 8 & 833.3 \\
\hline & Casero & 246.6 & 12 & 7.6 & 0.6 & 5.6 & 906.6 \\
\hline & Para asar & 330 & 15 & 10.6 & 0.6 & 1.6 & 850 \\
\hline Capistrano & Español & 374.6 & 14.5 & 12 & 4.1 & $\begin{array}{c}2.0 \\
6\end{array}$ & 910 \\
\hline \multirow[t]{2}{*}{ Great Value } & De pavo & 339.6 & 18 & 8.3 & 0 & 4.6 & 1260 \\
\hline & De cerdo & 370 & 21.3 & 11.3 & 0 & 3.6 & 1206.6 \\
\hline \multirow[t]{2}{*}{ FUD } & De pavo & 203.3 & 13.6 & 4.6 & 2 & 5 & 800 \\
\hline & De pavo (Cuida-t) & 203.3 & 13.6 & 4.6 & 2 & 5 & 800 \\
\hline $\begin{array}{l}\text { Chorizo Español } \\
\text { Alcalá }\end{array}$ & Cerdo & 333.3 & 10.6 & 11.6 & 1.6 & 5 & 500 \\
\hline Don Chema & Soya & 70 & 13 & 9 & 3 & 6 & 826 \\
\hline Soi-yah! & Vegetariano & 100 & 8.3 & 0.33 & 0 & 7.5 & 266 \\
\hline \multirow[t]{2}{*}{ Zwan } & Cerdo Cantimpalo & 290 & 14 & 12 & 1.3 & 2.6 & 653.3 \\
\hline & De Cerdo Español & 320 & 14.3 & 13.6 & 1.3 & 3 & 796.6 \\
\hline Hidalmex & Tipo español & 443 & 14.9 & 13.8 & 0.4 & 2.8 & 1217 \\
\hline
\end{tabular}

La OMS,${ }^{13}$ recomienda un consumo de $2 \mathrm{~g} / \mathrm{día}$ de sodio, ya que existe una fuerte relación entre el excesivo consumo de sal con los niveles elevados de presión arterial (PA), y el desarrollo de enfermedades cardiovasculares (ECV), ${ }^{14}$ por lo que el consumo de este producto debe ser moderado. 


\section{Conclusión}

Los chorizos evaluados se caracterizan por una alta densidad calórica (derivado de un alto contenido de proteína y grasa) y alto contenido de sodio, por lo que deben presentar en su etiquetado dos sellos de advertencia "Exceso de calorías" y "Exceso de sodio". Como excepción, los chorizos vegetarianos presentan bajo aporte calórico pero alto contenido en sodio, solo el chorizo Don Chema se sugiere muestre la advertencia en el etiquetado de "Exceso de grasa".

El contenido de fibra en algunos chorizos fue mayor al $2 \%$ que se recomienda, esto podría ser derivado de la utilización de soya en el procesamiento, sin embargo, no se mencionó en el etiquetado.

Finalmente se recomienda un consumo moderado de estos productos para evitar complicaciones o problemas de salud a futuro, como sobrepeso, obesidad y/o hipertensión, entre otras que pudieran estar relacionadas con el exceso de calorías, grasa saturada y sodio.

\section{Referencias}

[1] Jiménez M. Propuesta de un plan de mejora y control higiénico para productores de chorizo artesanal del Valle de Toluca en busca de una marca colectiva. [Tesis de maestría]. Toluca, México: Universidad Autónoma del Estado de México; 2013.

[2] Beauvois S. Sustitución de la grasa dorsal del cerdo por aceite de soya en la elaboración de chorizo tipo Antioqueño. [Tesis de pregrado]. Bogotá: Universidad de la Salle; 2016.

[3] Norma Oficial Mexicana NOM-122-SSA1-1991, Bienes y servicios. Productos de la carne. Productos cárnicos curados y cocidos, y curados emulsionados y cocidos. Especificaciones sanitarias. Disponible en

http://www.salud.gob.mx/unidades/cdi/nom/122ssa14.html

[4] Organización de las Naciones Unidas para la Agricultura y la Alimentación (FAO). Procesados de carnes. Fichas técnicas. [Internet]. 2014. (consultado el 10 de agosto de 2020). Disponible en: http://www.fao.org/3/a-au165s.pdf

[5] Norma Oficial Mexicana NOM-145-SSA1-1995, Productos Cárnicos Troceados y Curados. Productos Cárnicos Curados y Madurados. Disposiciones y Especificaciones Sanitarias. Disponible http://www.salud.gob.mx/unidades/cdi/nom/145ssa15.html

[6] Rocha-McGuire A. El uso de soya texturizada como extensor de productos cárnicos. Carnetec, artículos técnicos. [Internet]. 2010. (consultado el 9 de septiembre de 2020). Disponible en: www.carnetec.com

[7] Norma técnica NTON 03 103-16. Carne y productos cárnicos. Embutidos cárnicos. Características y especificaciones. Disponible
http://legislacion.asamblea.gob.ni/normaweb.nsf/(\$All)/6CB77 1737933A73A062582CC0063D361?OpenDocument

[8] Monckeberg B. La sal es indispensable para la vida, pero cuánta? Rev. Chil. Nutr. 2012; 39(4):192-195.

[9] Norma Oficial Mexicana NOM-051-SCFI/SSA1-2010, Especificaciones generales del etiquetado para alimentos y bebidas no alcohólicas preenvasados-Información comercial y sanitaria Disponible en: http://www.dof.gob.mx/normasOficiales/4010/seeco11_C/seec o11_C.htm

[10] Feiner G. Meat products handbook: Practical science and technology. CRC Press, Cambridge, England. 2006;73-74, 112113. ISBN: 1-85573-583-0.

[11] Marín-Martínez F. Estudio del efecto de la fibra dietética en la longaniza chilena: su incidencia en la calidad sensorial y en el comportamiento en la cocción. [Tesis de pregrado]. Santiago: Facultad de Cs. Químicas y Farmacéuticas, Universidad de Chile; 2008.

[12] Vargas-Velásquez C, López-Reinoso A, Flores-Artunduaga L. Evaluación de la concentración de nitratos/nitritos y cloruro de sodio en embutidos expendidos en la ciudad de Tarija. Rev Ventana Científica. 2014;1(7).

[13] Organización Mundial de la Salud (OMS). Ingesta de sodio en adultos y niños. [Internet]. 2019. (consultado el 16 de septiembre de 2020). Disponible en: https://apps.who.int/iris/bitstream/handle/10665/85224/WHO_ NMH_NHD_13.2_spa.pdf;jsessionid=CE4F0452D9E1B1E959 7D38076528C46B? sequence $=1$

[14] Valenzuela K, Atalah E. Estrategias globales para reducir el consumo de sal. ALAN. 2011;61(2):111-119. 